\title{
Letter
}

\section{Tunable light-matter hybridization in open organic microcavities}

Simon Betzold, Stefanie Herbst, Aurélien A. P. Trichet, Jason

M. Smith, Frank Würthner, Sven Höfling, and Christof Dietrich

ACS Photonics, Just Accepted Manuscript • DOI: 10.1021/acsphotonics.7b00552 • Publication Date (Web): 11 Oct 2017

Downloaded from http://pubs.acs.org on October 13, 2017

\section{Just Accepted}

"Just Accepted" manuscripts have been peer-reviewed and accepted for publication. They are posted online prior to technical editing, formatting for publication and author proofing. The American Chemical Society provides "Just Accepted" as a free service to the research community to expedite the dissemination of scientific material as soon as possible after acceptance. "Just Accepted" manuscripts appear in full in PDF format accompanied by an HTML abstract. "Just Accepted" manuscripts have been fully peer reviewed, but should not be considered the official version of record. They are accessible to all readers and citable by the Digital Object Identifier (DOI®). "Just Accepted" is an optional service offered to authors. Therefore, the "Just Accepted" Web site may not include all articles that will be published in the journal. After a manuscript is technically edited and formatted, it will be removed from the "Just Accepted" Web site and published as an ASAP article. Note that technical editing may introduce minor changes to the manuscript text and/or graphics which could affect content, and all legal disclaimers and ethical guidelines that apply to the journal pertain. ACS cannot be held responsible for errors or consequences arising from the use of information contained in these "Just Accepted" manuscripts. 


\section{Tunable light-matter hybridization in open organic microcavities}

Simon Betzold ${ }^{1}$, a) Stefanie Herbst ${ }^{2}$, Aurelien A. P. Trichet ${ }^{3}$, Jason M. Smith ${ }^{3}$, Frank Würthner ${ }^{2}$, Sven Höfling ${ }^{1,4}$, and Christof P. Dietrich ${ }^{1 \mathrm{~b}}$ )

${ }^{1}$ Technische Physik, University of Würzburg, Am Hubland, 97074 Würzburg, Germany

${ }^{2}$ Institut für Organische Chemie and Center for Nanosystems Chemistry, University of Würzburg, Am Hubland, 97974 Würzburg, Germany

${ }^{3}$ Department of Materials, University of Oxford, Parks Road, Oxford OX1 3PH, United Kingdom and

${ }^{4}$ SUPA, School of Physics and Astronomy, University of St Andrews, North Haugh, St Andrews KY16 9SS, United Kingdom

(Dated: 10 October 2017)

Open microcavities represent a versatile cavity design that allows the external control of internal properties such as cavity thickness and mode detuning without changing the key parameters of the cavity itself, rendering them particularly interesting for light-matter interaction experiments. Here, we demonstrate the tunability of an open microcavity with an embedded active organic layer providing parallel alignment of molecular transition dipole moments as well as strong self-absorption inside the cavity. By decreasing the cavity thickness, we observe a transition from the weak coupling regime into the strong coupling regime evidenced by the onset of avoided crossing behavior between involved modes. This change of coupling mechanism is shown for $2 \mathrm{D}$ (planar) as well as 0D (hemispherical) cavities.

Keywords: open microcavity, organic semiconductors, exciton-polaritons

Strong interactions between excitons and photons, e.g. in a semiconductor microcavity, lead to the formation of hybrid light-matter quasiparticles called cavity (exciton) polaritons ${ }^{1}$. In recent years, exciton-polaritons have attracted special attention in photonics research as their bosonic character features new emergent phenomena like non-equilibrium condensation ${ }^{2,3}$ or superfluidity ${ }^{4}$. Owing to the instability of Wannier-Mott excitons at elevated temperatures, many commonly used inorganic semiconductor materials do not support strong coupling at room temperature ${ }^{5}$. In contrast to this, Frenkel excitons, characteristic for organic semiconductors, possess much larger binding energies and are stable at room temperature, making polariton experiments at ambient air conditions feasible ${ }^{6}$. Organic materials further exhibit very large oscillator strengths and thus strongly interact with a cavity field easily. However, the implementation of organic semiconductors in optical microcavities is challenging because most organic materials are very sensitive to the depositing of semiconductor layers on top of them. Circumventing these issues, we use an open cavity system $^{7}$, which makes non-invasive investigation of the active material possible. During the last decade, several types of emitters have been studied towards single photon emission using tunable open cavity systems, including atoms $^{8}$, quantum dots ${ }^{9}$, single molecules ${ }^{10}$ or nitrogenvacancy centers in diamond ${ }^{11}$. In recent years, tunable microcavities are extensively used for strong lightmatter experiments on III-V microcavities ${ }^{12}$, monolayer

\footnotetext{
a)Electronic mail: simon.betzold@physik.uni-wuerzburg.de

b) Electronic mail: christof.dietrich@physik.uni-wuerzburg.de
}

materials ${ }^{13}$ or polymers ${ }^{14}$.

Here, we make use of a tunable open cavity system at ambient air conditions that comprises a bottom semiconductor distributed Bragg reflector (DBR) with a fluorescent emitter (MEH-PBI) on top and an upper DBR separated by a micrometer sized air gap (see Fig.1a for the cavity design). Both cavity halves are attached to piezo translation stages enabling the control of inter-mirror distance and mirror tilt. By decreasing the mirror separation by several micrometers, we observe a transition from a regime of weak interactions between emitter and cavity to a regime of strong light-matter interaction. This was demonstrated by the observation of pronounced mode anticrossing and theoretically confirmed by coupled oscillator calculations. The identification of this transition regime is particularly important for multi-lambda cavity systems such as the recently established laminated polariton microcavities ${ }^{15}$. By further introducing lensshape indentations into one of the mirrors, we observed mode discretization along the mode dispersion as result of in-plane confinement in both strong and weak coupling regimes rendering tunable cavities very interesting for more complex confinement potentials towards classical computation and quantum simulation experiments.

Our material of choice is the recently developed MEH$\mathrm{PBI}^{16}$. It belongs to the family of perylene bisimide dyes and is synthesized by tetra-substituting the bay position of the PBI core with four dendritic wedges with branched 2-ethylhexyl groups. This leads to a selfassembly through hydrogen bonds and $\pi$ - $\pi$-interactions into J-aggregates that further self-organize into liquidcrystalline domains. In this way, the molecular cores are oriented with their transition dipole moments parallel to each other and along the columnar axis. This situ- 

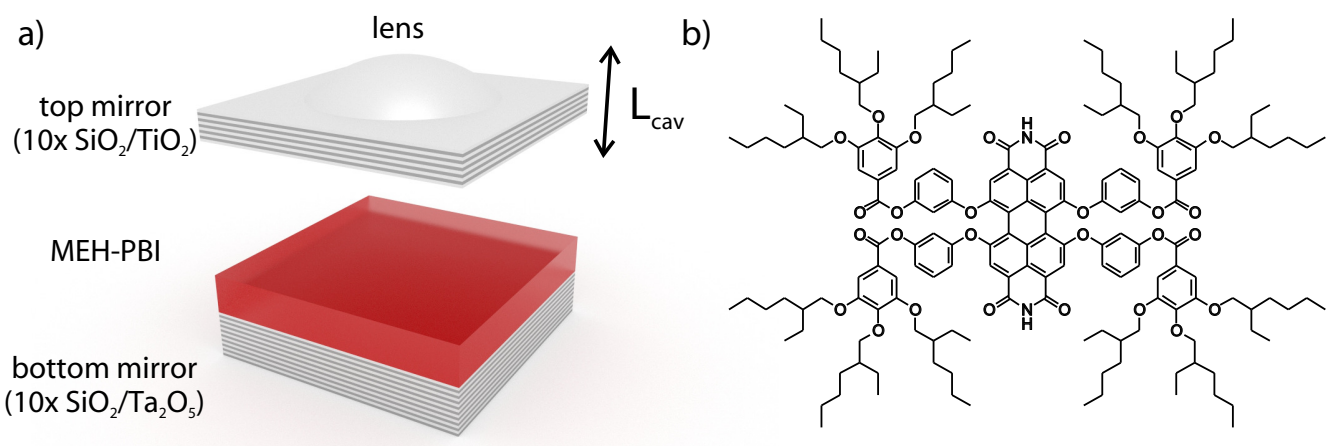

FIG. 1. a) Open cavity design: two DBR mirrors (composed of layer pairs of $\mathrm{SiO}_{2}$ and $\mathrm{TiO}_{2}$ ) are separated by an air gap. By attaching the mirrors to two individually translatable piezo stages, the cavity length $L_{\mathrm{Cav}}$ can be varied in a deterministic fashion. The incorporation of an active emitter material into the cavity makes the evolvement of strong interaction between active material and cavity photons possible. Further, the implementation of lens-shaped indentation into one of the cavity mirrors leads to an effective in-plane photonic confinement. b) The molecular structure of the organic compound MEH-PBI used as active fluorescent material in the open cavity.

ation is unprecedented in $\pi$-conjugated liquid crystalline (LC) phases and highly favorable with respect to coupling to the cavity light field. The absorption spectrum of MEH-PBI in its solid LC state (prepared by spin-casting from solution) is shown in Fig.2a (red line) exhibiting two excitonic maxima at $1.99 \mathrm{eV}(623 \mathrm{~nm})$ and $2.21 \mathrm{eV}$ $(561 \mathrm{~nm})$. The emission of solid-state MEH-PBI (yellow line) peaks at $1.93 \mathrm{eV}(642 \mathrm{~nm})$. We emphasize that there is pronounced spectral overlap between the absorption and emission spectrum which we have identified as being crucial for the establishment of strong light-matter interaction ${ }^{17,18}$.

DBRs were fabricated by means of ion beam deposition. Both mirrors are designed for maximum reflectivity close to the absorption maximum of MEH-PBI. While the bottom mirror is composed of 10 pairs of $\mathrm{SiO}_{2} / \mathrm{Ta}_{2} \mathrm{O}_{5}$ layers, the top mirror is composed of 10 pairs of $\mathrm{SiO}_{2} / \mathrm{TiO}_{2}$ in order to ensure both efficient light injection and outcoupling through the bottom mirror. Prior to coating, we have written lens-shaped indentations using ion beam milling ${ }^{19,20}$ into the top DBR substrate. The indentations have diameters ranging from 1 to $30 \mu \mathrm{m}$ and depths between 0.5 and $1.5 \mu \mathrm{m}$. One key figure for spherical resonators is the radius of curvature $R_{\mathrm{C}}$ that in our case ranges from 5 to $250 \mu \mathrm{m}$. The organic layer is spin-coated from solution (solved in carbon tetrachloride) on top of the bottom mirror with a thicknesses of about $300 \mathrm{~nm}$.

The two cavity parts were each attached to a threeaxis nanopositioner (piezo actuators) allowing in-plane translation as well as change of mirror distance which is equivalent to a change of spectral detuning. In addition to that, one of the two nanopositioners is equipped with goniometers accounting for eventual tilting of the mirrors with respect to each other and the optical beam axis. The open microcavity emission was excited through the bottom mirror by the $532 \mathrm{~nm}$-line of a continuous-wave diode laser that is resonant with the second Bragg minimum of the top mirror, collected in reflection geometry (again through the bottom mirror) and monitored onto the entrance slit of a spectrometer with $0.06 \mathrm{~nm}$ spectral resolution able to resolve mode quality factors up to 10000. We have further performed Fourier imaging by using a high-NA objective $(50 \mathrm{x}, \mathrm{NA}=0.42)$ in front of the microcavity sample and an additional Fourier lens in the beam path in order to project the angle-dependent emission onto the entrance slit. The absorption of the active material was measured by transmission experiments using a collimated white light beam.

Fig.2b-d shows the emission spectrum of an open MEH-PBI microcavity for three different mirror separations measured on a planar spot of the microcavity. For the largest separation shown $(20 \mu \mathrm{m}$ in Fig.2b), the microcavity emission mainly follows the same intensity profile as measured outside the cavity for MEH-PBI (yellow line in Fig.2a). However, emission is also funneled into longitudinal cavity modes that occur on top of the emission intensity profile. All cavity modes appear to be spectrally asymmetric as result of image binning along the angular axis. Hence, we attribute the high-energy shoulder of the cavity modes to the angle-dependent mode dispersions. As the majority of cavity modes occurs in the emissive spectral region of MEH-PBI and emission is largely suppressed in the absorptive region of $\mathrm{MEH}-\mathrm{PBI}$, we assume to be dominated here by the regime of weak light-matter interactions.

In Fig.2b, the energy of maximum intensity of the microcavity emission seems to be shifted with respect to the emission maximum of MEH-PBI owing to the onset of self-absorption within the cavity. Decreasing the mirror separation to about $8 \mu \mathrm{m}$ (see Fig.2c), those effects are still present. However, the free spectral range is consid- 


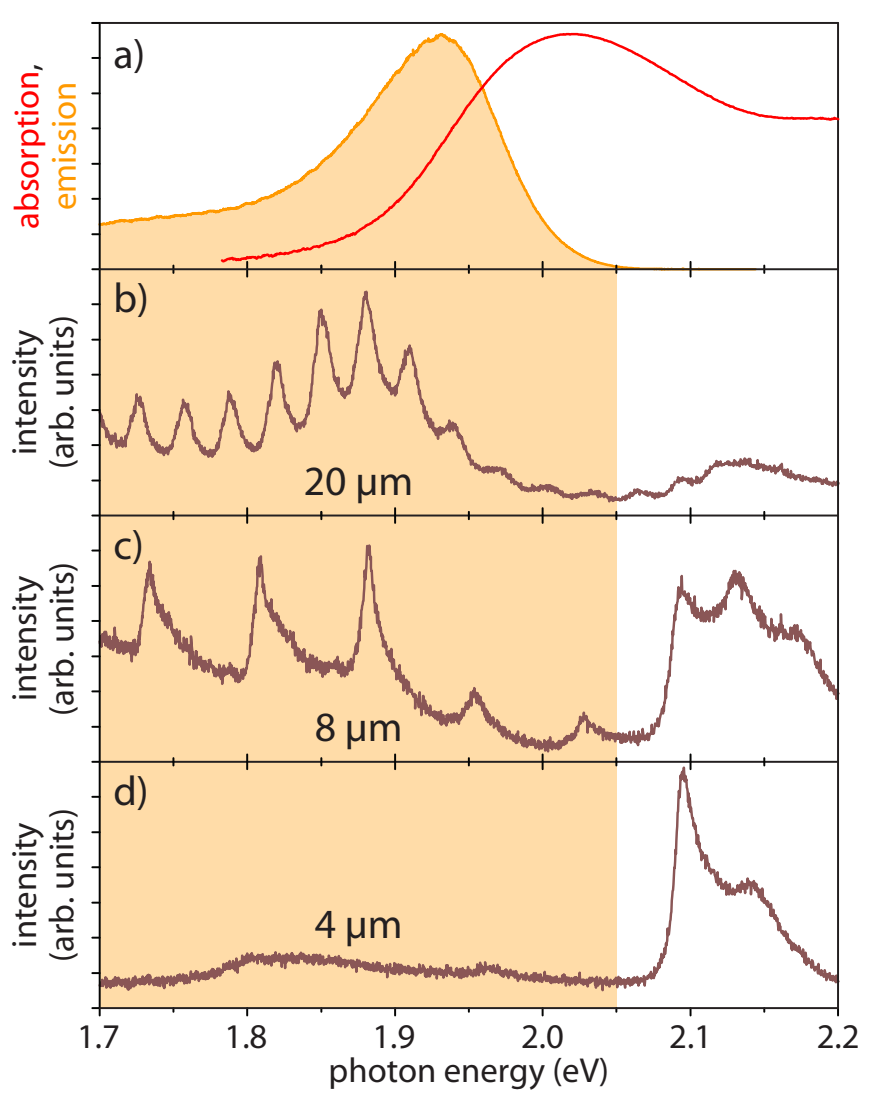

FIG. 2. a) Emission (yellow line) and absorption (red line) spectrum of MEH-PBI. b-d) Open microcavity emission for different mirror separations: $20 \mu \mathrm{m}$ (b), $8 \mu \mathrm{m}$ (c) and $4 \mu \mathrm{m}$ (d). The yellow area highlights the spectral region of MEHPBI emission.

erably decreased as result of the decreased cavity length and, more importantly, emission in the absorptive region of MEH-PBI becomes more and more apparent. If we decrease the mirror separation further (to about $4 \mu \mathrm{m}$, see Fig.2d), emission in the emissive region of MEH-PBI is almost entirely suppressed and only peaks in the absorptive region. We attribute this shift to a transition of the microcavity to a change of the interaction regime from mainly weak to predominantly strong interactions between light and matter. The latter is fully governed by the excitonic transitions of the active organic material and the cavity field leading to the formation of polariton modes.

In order to confirm this hypothesis, we have performed a fine-tuned thickness-dependent measurement of the microcavity emission in the close proximity regime. This can be seen Fig.3c (all spectra are shown only along the normal direction, $k_{x, y}=0$ ) where we have changed the applied bias on the piezo actuators and, in this way, tuned several apparent modes across the excitonic range of MEH-PBI. It is obvious from Fig.3c, that the observed modes do not linearly shift with increased cavity thickness but rather show deflective behavior - the fingerprint for strong coupling between cavity photons and MEH-
PBI excitons. This is in accordance with transfer matrix calculations that we have performed for an active (see Fig.3a) and a passive microcavity (shown in Fig.3b). As a reference experiment, we have carried out reflectivity measurements in the large mirror separation regime (please see Fig.3e) for an empty microcavity where we tuned the cavity modes across the excitonic transitions showing no effect on the spectral position of cavity modes whatsoever. We have subsequently extracted the maxima of all observed modes from Fig.3c and plotted them in Fig.3d together with a coupled oscillator fit $^{15}$ (red lines) taking into account the uncoupled cavity field modes (orange lines) as well as the two main excitonic resonances (gray lines) revealing remarkable agreement between experiment and theory. From the fit, we extract excitonphoton coupling strengths of $114 \mathrm{meV}$ and $86 \mathrm{meV}$ (for the two excitonic transitions, respectively). Those values are in the same range as previously determined values for exciton-photon systems with the same active material ${ }^{18}$. The difference between the extracted values can easily be explained by the different cavity designs involving different cavity thicknesses.

Also the angle-resolved emission of the microcavity from Fig.3c shows those peculiar features that would only be expected in the strong light-matter interaction regime. It can be seen in Fig.4a for a thickness of around $5.5 \mu \mathrm{m}$, the respective spectrum at $k_{x, y}=0$ is indicated by the vertical dotted white line in Fig.3c. It shows several modes that blueshift with increasing wavevector (equivalent to an increasing emission angle) due to their dispersion. We find the lower two modes (M1-M2) with a large mode separation whereas the middle two modes (M2-M3) show a much smaller spectral distance. The upper two modes (M3-M4) then again exhibit larger mode spacing. It seems that those modes that lie spectrally within the two excitonic resonances are pushed together. However, it is evident that there is a clear interaction between the cavity field and the excitonic reservoir of MEH-PBI. Similar to Fig.3c, we have also extracted the angle-resolved mode maxima and plotted them in Fig.4b together with a coupled oscillator model using exactly the same parameters as in Fig.3d again revealing very good correlation.

While the planar cavity design provides very good mode confinement along the normal (out-of-plane) direction, it suffers from photonic losses within the layer plane. Those losses can largely be suppressed by introducing photonic traps along the layer plane. Here, we achieve three-dimensional confinement by writing lensshaped indentations into the top mirror prior to coating. A one-dimensional cut through a three-dimensional surface profile (measured by means of atomic force microscopy, AFM) of one of the written concave lenses after coating can be seen in Fig.5a. The profile indicates that the shape of the lens is a section of a sphere theoretically leading to the formation of hemispherical resonator modes. This is particularly important as, according to resonator theory, hemispherical cavities are only stable as long as the cavity length is smaller than the radius 

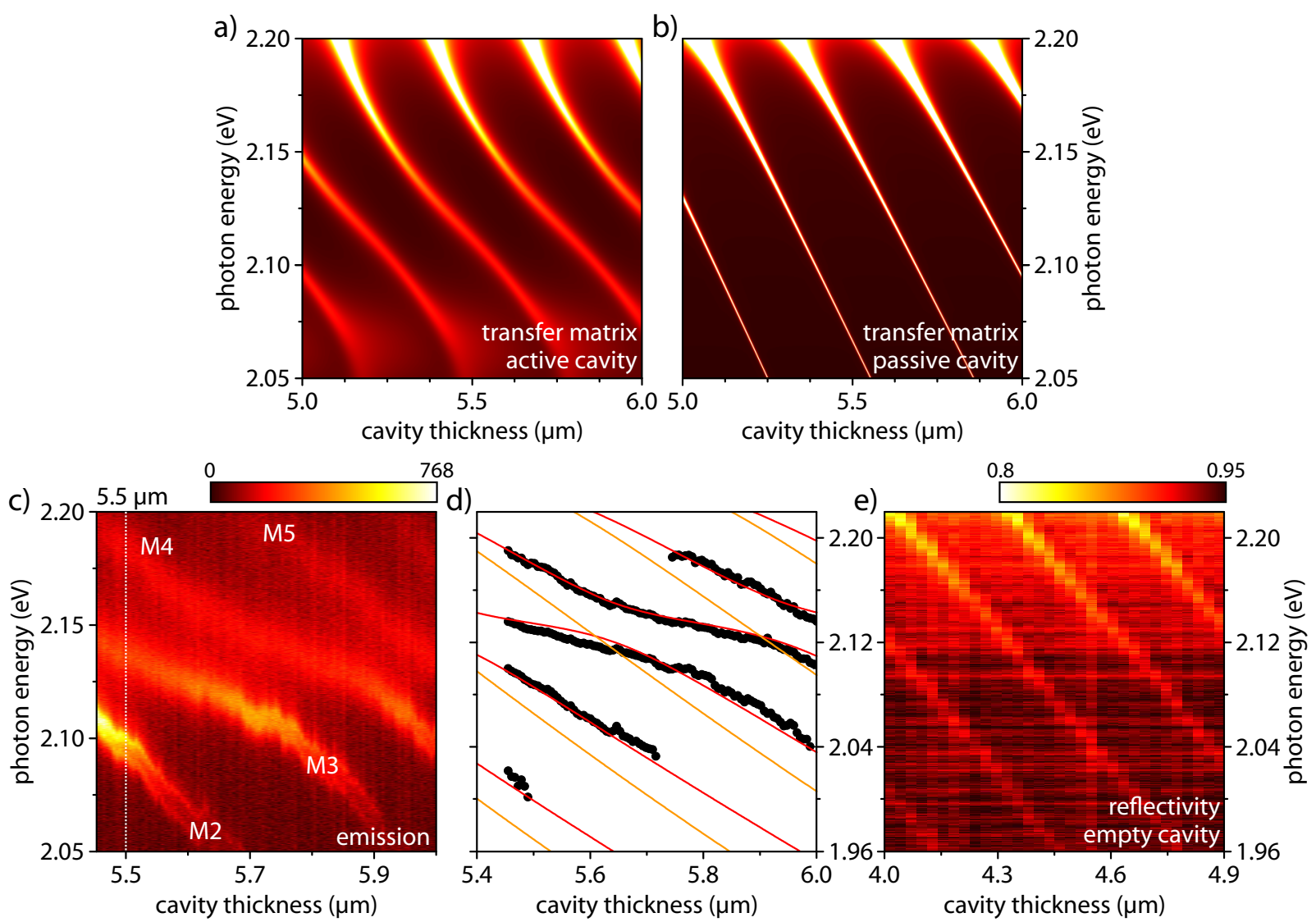

FIG. 3. a,b) Thickness-dependent reflectivity from transfer matrix calculations for an active (a) and a passive (b) open MEH-PBI microcavity. The thickness of the MEH-PBI film was set to $300 \mathrm{~nm}$ in both cases. c) Thickness-dependent normal emission $\left(k_{x, y}=0\right)$ of an open microcavity filled with MEH-PBI. d) Extracted emission resonances (black circles, from (c)) and simulated polariton modes (red solid lines). The respective uncoupled cavity modes are shown as solid orange lines. Please note the different photon energy scale compared to (c). e) Fine-tuned, thickness-dependent reflectivity of an empty microcavity.

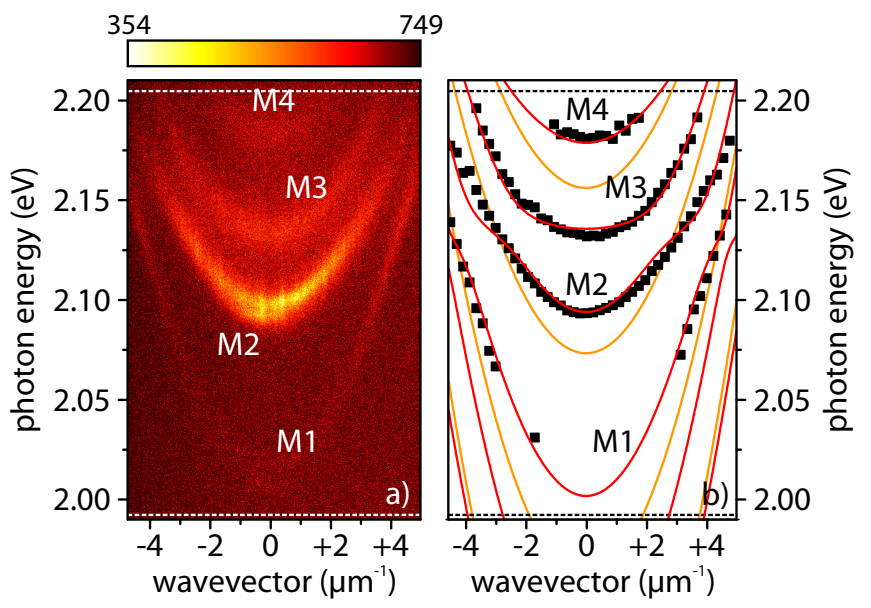

FIG. 4. a) Angle-resolved emission of an open microcavity filled with MEH-PBI and tuned to $L_{\mathrm{Cav}}=5.5 \mu \mathrm{m}$. b) Extracted emission resonances (black squares, from a) and simulated polariton modes (red solid lines). The respective uncoupled exciton (photon) modes are shown as dashed gray (solid orange) lines. of curvature $R_{\mathrm{C}}$ of the concave mirror ${ }^{21}$. In order to determine $R_{\mathrm{C}}$ for all written indentations and to verify the stability criterion, we have fitted the AFM images with a spherical formula and derived values for $R_{\mathrm{C}}$ ranging from 10 to $250 \mu \mathrm{m}$. We have further recorded the emission of hemispherical microcavities filled with MEHPBI along the normal direction and determined the mode quality factor $Q$ of longitudinal modes in dependence of $R_{\mathrm{C}}$ for a constant cavity thickness of $L_{\mathrm{Cav}}=10 \mu \mathrm{m}$ that can be seen in Fig.5b. A clear maximum is obvious at $R_{\mathrm{C}}=30 \mu \mathrm{m}$. While the drop in $Q$ at smaller radii of curvature is caused by approaching the stability condition of the hemispherical resonator, we attribute the decrease of quality factors at higher $R_{\mathrm{C}}$ to an increase of photonic losses within the layer plane.

Fig.5c shows an angle-resolved emission pattern of an open MEH-PBI microcavity recorded on the spatial position of a concave lens (the pattern covers most of the emissive spectral region of MEH-PBI). The cavity thickness in this case is about $8 \mu \mathrm{m}$. In contrast to the planar case (Fig2c), the emission pattern shows the formation of additional dispersion-less transversal cavity modes as result of the three-dimensional mode confinement achieved 

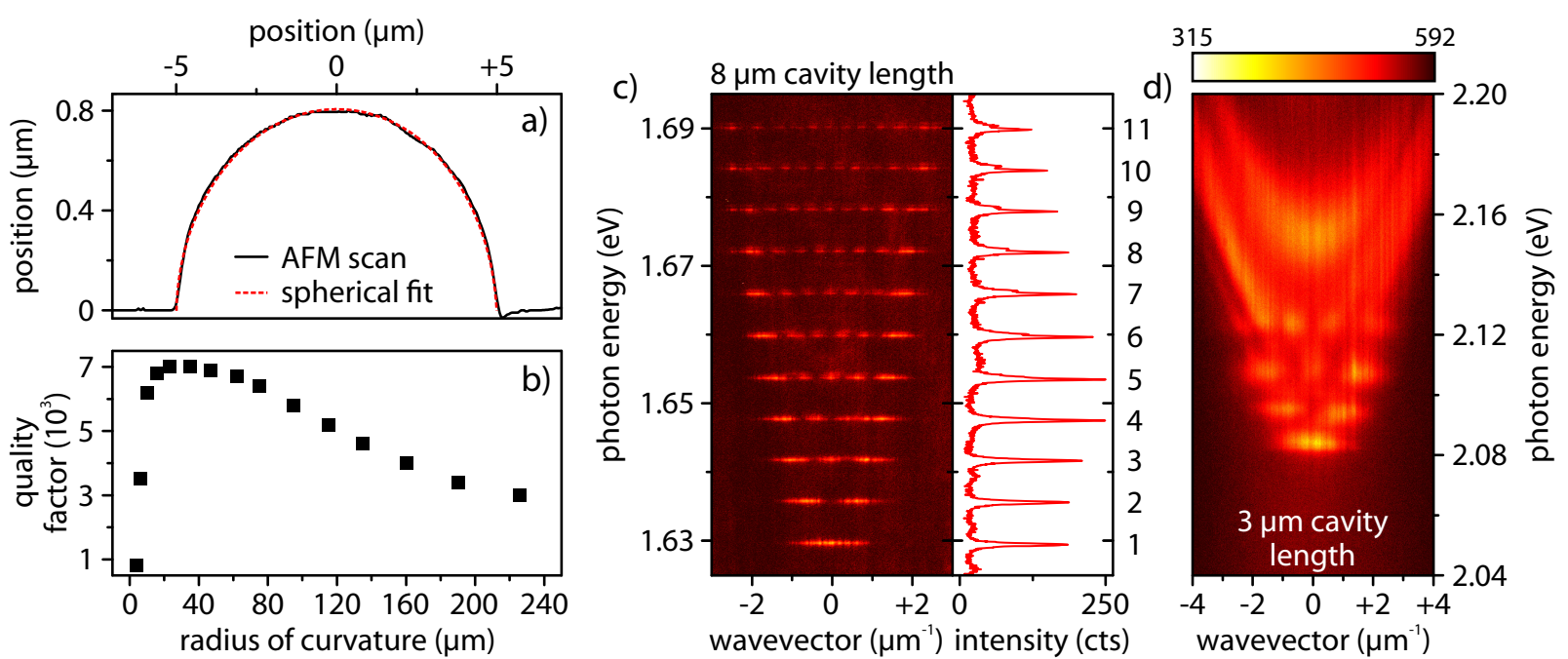

FIG. 5. a) AFM linescan trough a lens-shaped indentation written into a $\mathrm{SiO}_{2}$ substrate by ion beam milling and coated by a DBR (10 pairs of $\mathrm{SiO}_{2} / \mathrm{TiO}_{2}$ ). b) Mode quality factors $Q$ of uncoupled cavity modes (transversal mode ground state) versus radius of curvature $R_{\mathrm{C}}$ (for a constant cavity thickness of $L_{\mathrm{Cav}}=10 \mu \mathrm{m}$ ). c,d) Angle-resolved emission patterns of an open MEH-PBI microcavity measured on the position of a concave lens for a cavity length of $8 \mu \mathrm{m}$ (c) and $3 \mu \mathrm{m}$ (d). Numbers in c) indicate the respective transversal mode number.

by the concave lens. Modes can clearly be observed up to high transversal mode numbers (e.g. up to $m=11$ in Fig.5c) indicating a photonic confinement potential of at least $40 \mathrm{meV}$. The transversal mode patterns are of Laguerre-Gaussian-type (as expected in systems with cylindrical symmetry). The mode spacing is further constant throughout the spectrum in accordance with hemispherical resonator theory ${ }^{21}$. Consecutively, we have decreased the mirror separation below a value that we have identified as transition point into the regime of strong light-matter interaction for a planar microcavity. The respective emission pattern for $L_{\text {Cav }}=3 \mu \mathrm{m}$ can be seen in Fig.5d. Similar to what was observed in Fig.2, the microcavity emission only appears in the absorptive spectral region of MEH-PBI. Fig.5d shows emission from the planar background around the irradiated lens but, more importantly, emission into dispersion-less transversal modes (red-shifted by the confinement potential with respect to the planar background) that can be addressed as radiative decay of $0 \mathrm{D}$ exciton-polaritons formed by the strong interaction between MEH-PBI excitons and the cavity light field being three-dimensionally confined in the lens area.

In conclusion, we have demonstrated the advantages of an open cavity approach for the investigation of coupled exciton-photon systems at ambient air conditions facilitating the external tuning of the mirror separation and thereby enabling deterministic switching of light-matter coupling from a predominantly weakly to a predominantly strongly interaction exciton-photon system. In our case, we have made use of the peculiar optical properties of the newly developed organic LC compound MEHPBI that exhibits parallel arrangement of molecular transition dipole moments and self-absorption strongly sup- porting light-matter interaction. Microcavity emission was recorded for planar and hemispherical resonators unambiguously showing the transition from weak to strong coupling by reducing the mirror separation. When increasing the quantum yield of the active material, its concentration as well as layer thickness, we believe that this open organic cavity system can readily be brought into the non-linear regime of polariton condensation, where it displays an externally tunable, low-threshold coherent light source at room temperature. Furthermore, the implementation of a deterministic polariton trap (using lenses in the upper mirror) represents the basis for more complex photonic potentials (such as waveguide structures or lattices) towards possible classical computation and quantum simulation experiments.

We thank M. Emmerling for support during ion beam milling, H. Bostelmann-Arp for help during the analysis of AFM data and M. Dusel for image preparation. Sv.H. acknowledges funding by the EPSRC "Hybrid Polaritonics" Grant (EP/M025330/1), F.W. further thanks the Deutsche Forschungsgemeinschaft (DFG) for financial support (WU317/18-1). J.S. and A.A.P.T. thank the Leverhulme Trust (RPG-2012-526) and the UK Engineering and Physical Sciences Research Council (EP/M013243/1) for financial support.

\section{REFERENCES}

${ }^{1}$ A.V. Kavokin and G. Malpuech, Cavity Polaritons (Academic Press, 2003).

${ }^{2}$ J. Kasprzak, M. Richard, S. Kundermann, A. Baas, P. Jeambrun, J.M.J. Keeling, F.M. Marchetti, M.H. Szymanska, R. Andre, J.L. Staehli, V. Savona, P.B. Littlewood, B. Deveaud, and L.S. Dang, Nature 443, 409 (2006). 
${ }^{3}$ R. Balili, V. Hartwell, D. Snoke, L. Pfeiffer, and K. West, Science 316, 1007 (2007)

${ }^{4}$ A. Amo, J. Lefrere, S. Pigeon, C. Adrados, C. Ciuti, I. Carusotto, R. Houdré, E. Giacobino and A. Bramati, Nat. Phys. 5, 805 (2009).

${ }^{5}$ T. Guillet, and C. Brimont, Comp. Rend. Phys. 17, 946 (2016).

${ }^{6}$ D.G. Lidzey, D.D.C. Bradley, M.S. Skolnick, T. Virgili, S. Walker and D.M. Whittaker, Nature 395, 53 (1998).

${ }^{7}$ M. Trupke, E.A. Hinds, S. Eriksson, E.A. Curtis, Z. Moktadir, E. Kukharenka, and M. Kraft, Appl. Phys. Lett. 87, 211106 (2005).

${ }^{8}$ M. Trupke, J. Goldwin, B. Darquie, G. Dutier, S. Eriksson, J. Ashmore and E. A. Hinds, Phys. Rev. Lett. 99, 063601 (2007).

${ }^{9}$ A. Muller, E.B. Flagg, M. Metcalfe, J. Lawall and G.S. Solomon, Appl. Phys. Lett. 95, 173101 (2009).

${ }^{10}$ C. Toninelli, Y. Delley, T. Stöferle, A. Renn, S. Götzinger, and V. Sandoghdar, Appl. Phys. Lett. 97, 021107 (2010).

${ }^{11}$ R. Albrecht, A. Bommer, C. Deutsch, J. Reichel and C. Becher, Phys. Rev. Lett. 110, 243602 (2013).

${ }^{12}$ S. Dufferwiel, F. Fras, A. Trichet, P.M. Walker, F. Li, L. Giriunas, M.N. Makhonin, L.R. Wilson, J.M. Smith, E. Clarke, M.S. Skolnick, and D.N. Krizhanovskii, Appl. Phys. Lett. 104, 192107 (2014).

${ }^{13}$ S. Schwarz, S. Dufferwiel, P.M. Walker, F. Withers, A.A.P.
Trichet, M. Sich, F. Li, E.A. Chekhovich, D.N. Borisenko, N.N. Kolesnikov, K.S. Novoselov, M.S. Skolnick, J.M. Smith, D.N. Krizhanovskii and A. I. Tartakovskii, Nano Lett. 14, 7003 (2014). ${ }^{14}$ D. Urbonas, T. Stöferle, F. Scafirimuto, U. Scherf, and R.F. Mahrt, ACS Photonics 3, 1542 (2016).

${ }^{15}$ C.P. Dietrich, A. Steude, L. Tropf, M. Schubert, N.M. Kronenberg, K. Ostermann, S. Höfling, and M.C. Gather, Sci. Adv. 2, e1600666 (2016).

${ }^{16}$ S. Herbst, B. Soberats, P. Leowanawat, M. Lehmann, and F. Würthner, Angew. Chem. Int. Ed. 56, 2162 (2017).

${ }^{17}$ C.P. Dietrich, S. Höfling, and M.C. Gather, Appl. Phys. Lett. 105, 233702 (2014).

${ }^{18}$ L. Tropf, C.P. Dietrich, S. Herbst, A.P. Kanibolotsky, P. Skabara, F. Würthner, I.D.W. Samuel, M.C. Gather, and S. Höfling, Appl. Phys. Lett., accepted (2017).

${ }^{19}$ P.R. Dolan, G.M. Hughes, F. Grazioso, B.R. Patton, and J.M. Smith, Opt. Lett. 35, 3556 (2010).

${ }^{20}$ A.A.P. Trichet, P.R. Dolan, D.M. Coles, G.M. Hughes, and J.M. Smith, Opt. Expr. 23, 17205 (2015).

${ }^{21}$ A.E. Siegman, Lasers, University Science Books (Mill Valley, 1986). 


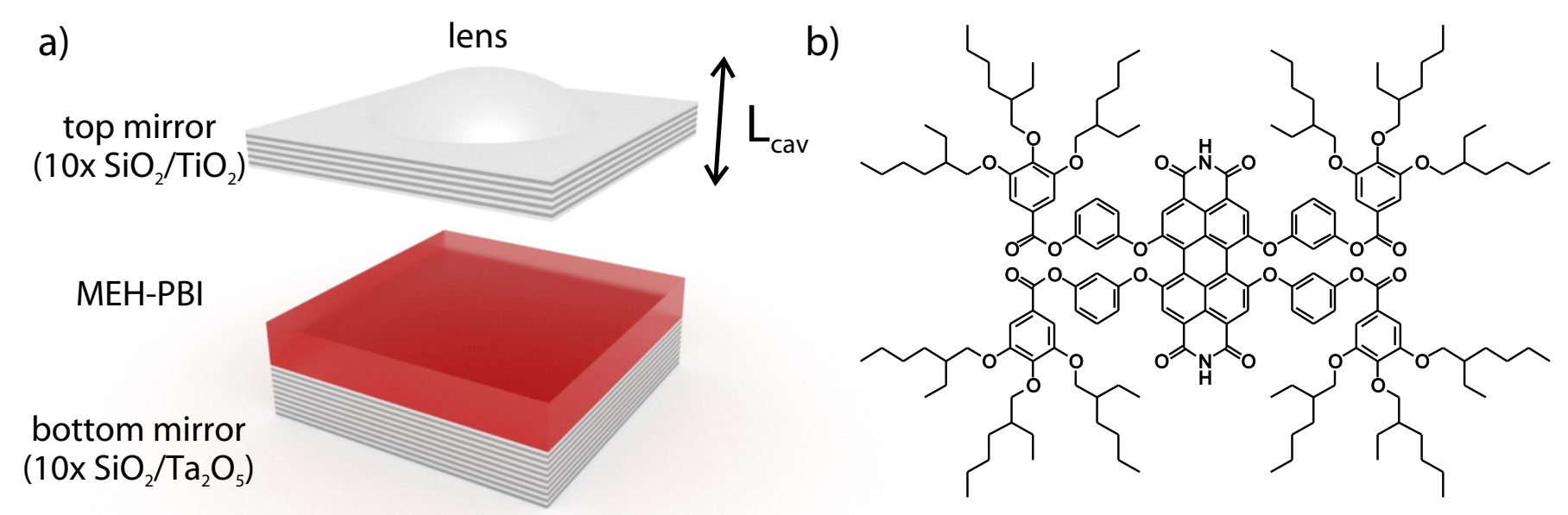




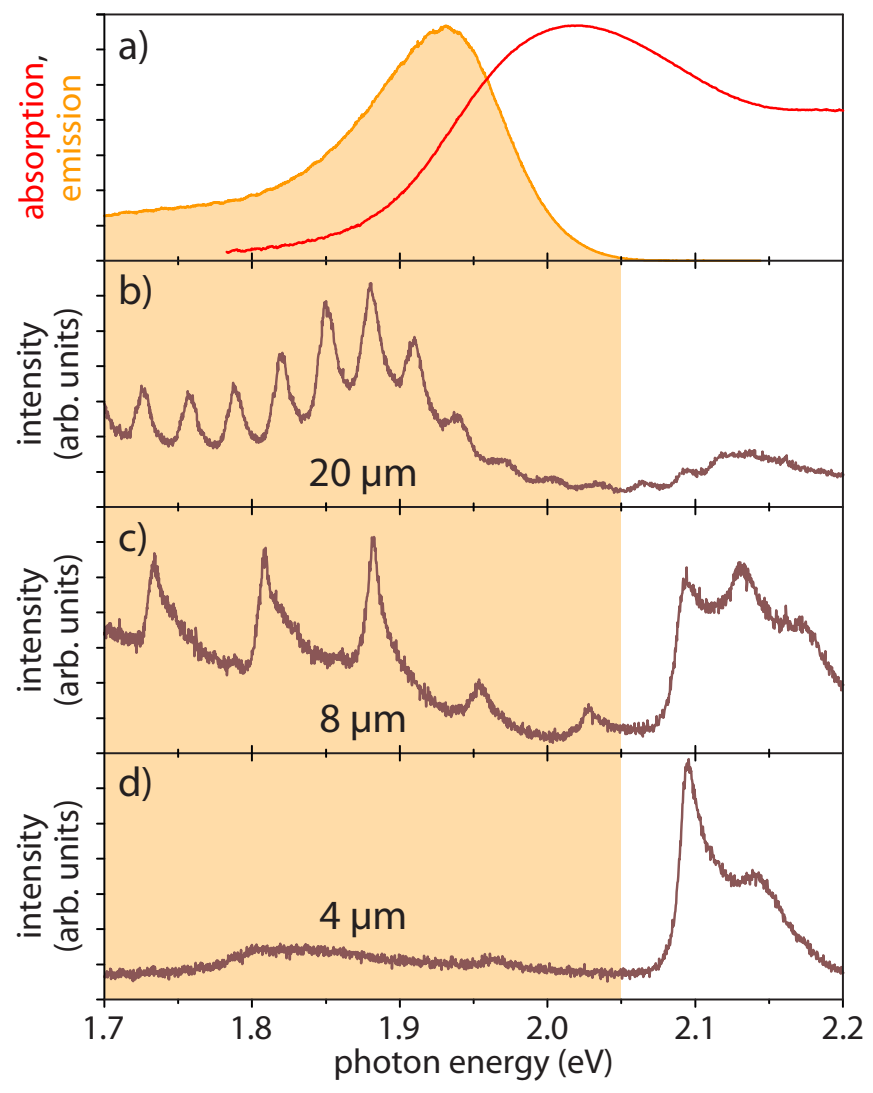

ACS Paragon Plus Environment 

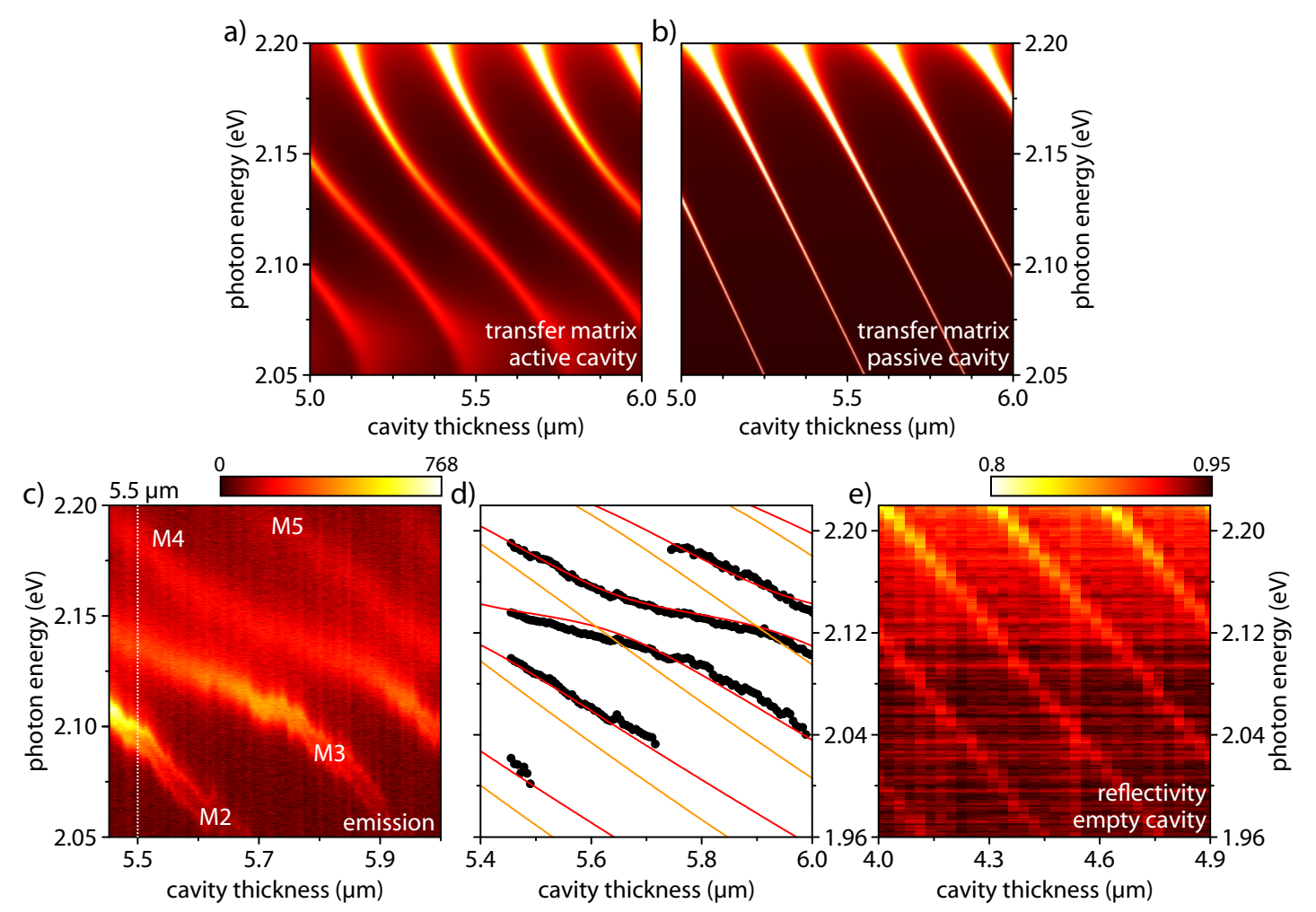

ACS Paragon Plus Environment 


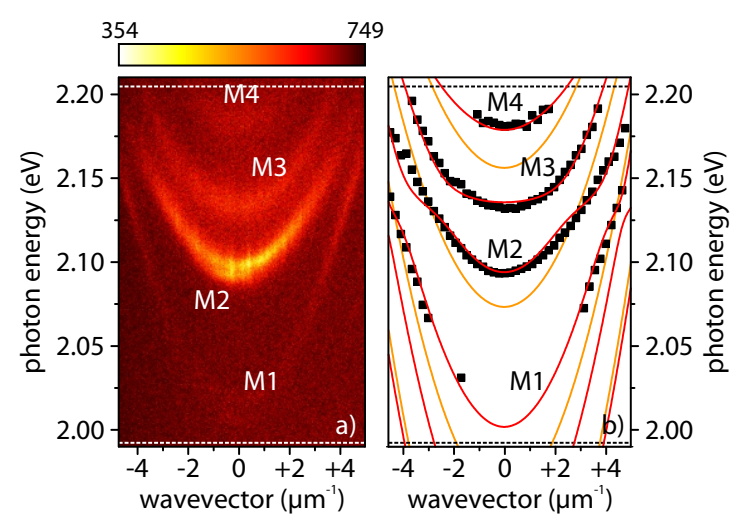

ACS Paragon Plus Environment 


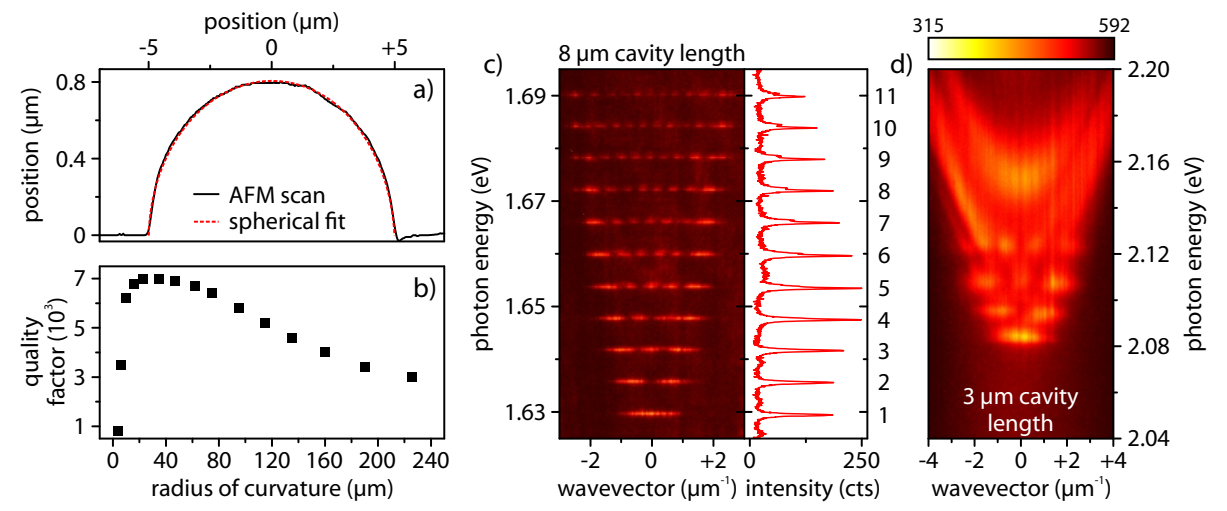




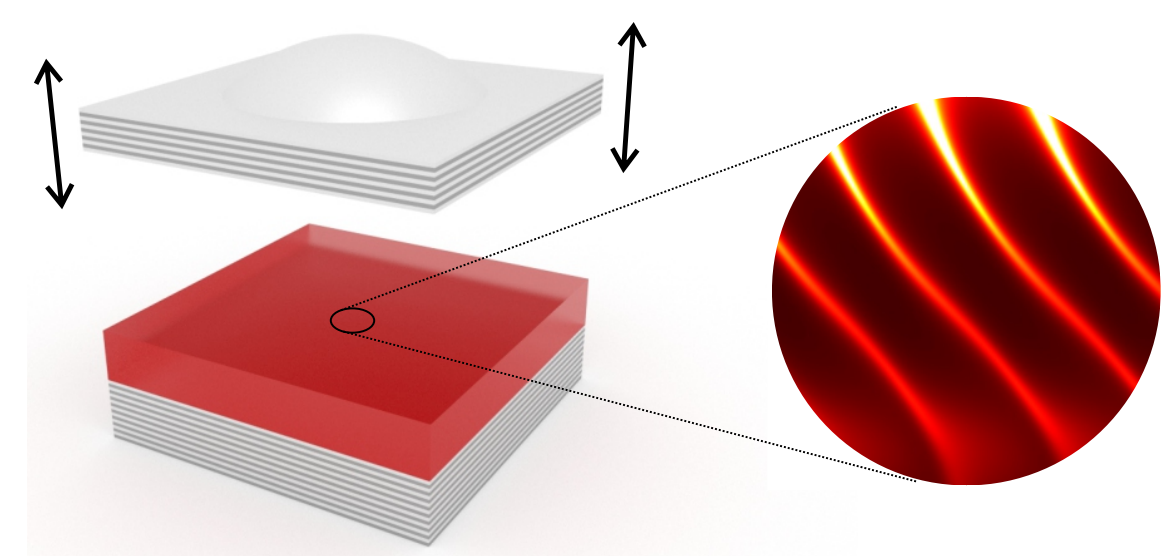

ACS Paragon Plus Environment 